\title{
Big Data Pathway for the Future Talent
}

by Evan Stubbs and Chami Akmeemana

\section{Executive Summary:}

Business analytics enables information advantage. This information advantage will be a significant source of competitive differentiation through the 21st century. Unfortunately, labor market shortages will prevent many organizations from realizing this advantage. Realizing value through business analytics will involve acquiring and retaining data scientists, experts in insight generation, and value architects, and experts in change management. In a time of exponential data growth, organizations that understand how best to identify, connect with, and develop the right skills will increasingly differentiate themselves from their peers.

\section{Information Advantage: The 21st Century Differentiator KEY POINTS:}

- Big data represents one of the greatest opportunities and challenges in recent history

- There are severe labor market imbalances that will prevent many organizations from realizing these opportunities

- Most organizations will need more than data scientists to succeed

The race for an information advantage is on. Data volumes grow on a daily basis. According to IDC, "in 2011, the amount of information created and replicated will surpass 1.8 zettabytes (1.8 trillion gigabytes), growing by a factor of nine in just five years. That's nearly as many bits of information in the digital universe as stars in the physical universe."

Unfortunately, the supply of skilled analytics professionals is tightening. McKinsey, a consultancy, suggests that by 2018 the US alone will face a shortage of up to 190,000 people with deep analytics skills and 1.5 million managers and analysts with the know-how to use big data to make effective decisions.

Gartner, a research firm, suggests a direr conclusion: by 2015, more than 4.4 million jobs will be required to support big data initiatives. Unfortunately, less than a third of these will be filled due to a global resource shortage.

Analytics may well become the engine of the 21 st century economy. The Economist suggests that these shifts are so significant that organizations will need a radically different approach to how they harness their information. Some commentators even go so far as to suggest that today's big data will just be tomorrow's normal data.
McKinsey estimates that services enabled by personal-location data might allow consumers to realize $\$ 600$ billion in consumer surplus. A recent survey of Federal government IT decision makers suggested that big data might generate as much as $\$ 380$ billion in savings.

At its core, business analytics is about change. Analyzing information creates insight - insight that supports a reason for change. Knowing how to make that change happen such that it links information to value is the root of future competitive advantage. However, without the horsepower to create this insight and manage this change, many organizations will be left stranded.

The field has many barriers to entry and is filled with technical jargon, like "dark data", "big data", "machine learning", and "experimental design". While best practices exist, the field is still a nascent one. Normally, the solution would be simple: hire experienced people. Unfortunately, it's hard to find the perfect person and without the right combination of skills, it is far easier to fail in business analytics than it is to succeed.

The best kept secret in the industry is this: when it comes to developing competitive advantage through business analytics, pure analytics is only half the picture. And without having access to the missing half, it's dangerously easy to lose time and money.

Data scientists are important. So is having access to high quality data. Together, they are still not enough to guarantee value from information. Rather than trying to hire one set of skills, most organizations benefit from developing two different types of capabilities.

Data scientists are great at generating insight. They know how to solve hard problems. Their limitations are often in getting organizations to act on that insight. Getting to that point requires someone different: the value architect, an expert in managing change and quantifying value. Where a data scientist generates the answer, the value architect is an expert in getting an organization to act on that answer.

Together, these different but aligned capabilities translate insight into action. Analytical innovators are four times more likely to "agree" or "strongly agree" that analytics has shifted power to those with the insight rather than those with the opinions in their organizations compared to analytically challenged companies. They understand the importance of developing the right culture, delivering real results, and the need to change.

These skills make the difference between knowing what to do and actually doing it. And by acquiring and retaining the right breadth of skills, organizations create an information advantage. By following 
the right approach, understanding what to look for, and asking the right questions, organizations substantially increase their odds of success in business analytics.

\section{Extracting Insight from Information: The Data Scientist KEY POINTS:}

- Data scientists, while strong in generating insight, are often weaker in getting organizations to act on that insight

- The best data scientists draw their experience from multiple disciplines, are comfortable working with all aspects of big data, and bring a scientific mindset to analytics

- Recruiting highly talented data scientists often goes beyond pure remuneration and requires a good understanding of what's on offer elsewhere

Using data to answer hard questions is not new. Whether they're called quants, statisticians, analysts, or the now quaint term "computers", using data to answer questions has a long pedigree, even going as far back as the turn of the 20th century. What is different is the breadth of skills held by a modern data scientist.

Unlike a traditional analyst, the data scientist brings more to bear than just mathematical or analytical skills. The need for good communication skills is well understood. While this is equally true for data scientists, there are specific differences between a data scientist and a traditional analyst.

Firstly, the data scientists bring a scientific mindset to answering problems. A good data scientist is an expert in simplification, reducing complex problems down to their component parts. Each of these forms a testable hypothesis with well-defined answers. They get to the heart of the problem and their interest is in solving the problem, not creating novel theories or pure research and develop-

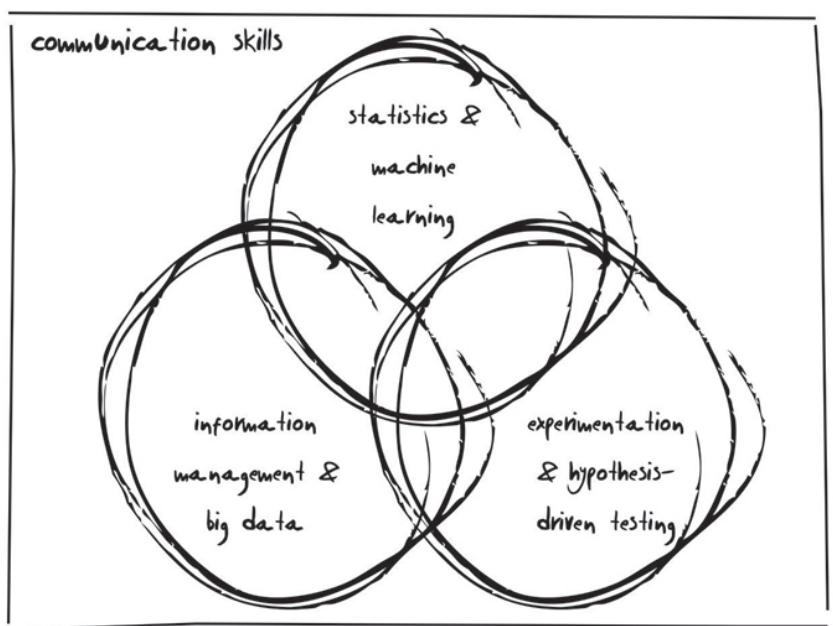

ment. Their defining trait is curiosity, not acceptance; when presented with a contradiction, their chief interest is "why".

Secondly, they have the ability to work with big data in its broadest sense. While they're comfortable with large amounts of data, they're equally at home with unstructured and high-velocity data. Being able to handle petabytes of data is the minimum benchmark; an excellent data scientist is also comfortable working with textbased data and real-time data. Presented with an apparently insurmountable data challenge, their main interest will be in overcoming it, not accepting it.

Finally, a true data scientist draws their analytical knowledge from multiple disciplines. More than a pure statistician, they may be equally comfortable working across linguistics, graph theory, and unsupervised machine learning. While it's probably impossible to create a checklist and it's rare to come across true polymaths, data scientists create novel solutions through being able to draw from many academic disciplines. Predicting churn in telecommunications might not seem similar to managing mortality rates in the medical professional. A good data scientist will, however, immediately see the connection.

True data scientists are a rare breed. While many people will claim the title over the next few years, few will have the skills to drive novel or innovative insights within the organizations they work with. Because of this, most organizations will face two challenges.

The first is simply finding real data scientists. While it's fairly easy to negotiate a title, it's extremely difficult to become truly proficient in a highly cross-disciplinary profession. Given increasing demand for data scientists, most organizations will struggle to tell the difference between real data scientists and traditional analysts. Being unable to differentiate carries real costs; organizations end up over-paying and carrying significant opportunity costs. By hiring the wrong person they'll lose time, time that cannot be reclaimed.

Telling the difference between a competent data scientist and an aspirational spreadsheet jockey isn't always easy. References, experience, and history are all strong indicators. However, there's one thing that's even stronger: the ability to draw on a global network. The best people are already known by their peers whether it's through online competitions run through Kaggle or through professional relationships. Often, the fastest way to enter this network is to connect with partners who already have the necessary relationships.

Hiring the right person is only the start. Once hired, many organizations will discover to their dismay that data scientists seem fickle. Many data scientists will look elsewhere if they are not presented with problems that challenge them, a career path that fulfills them, and a salary that satisfies them. Given the ongoing labor shortage, 
this should not be surprising; it's a rare person who won't take the best offer available to them.

What may be surprising is that not all data scientists are motivated by remuneration. While money is always a factor, many data scientists are equally motivated by the freedom to experiment, the opportunity to work on challenging problems, and the chance to build something, not just advise someone. Hiring and retaining a skilled data scientist usually involves more than just negotiating a competitive salary. Without sufficient challenge, most data scientists will eventually look for greener pastures.

The core of data science will change little over the next decade. The problems it answers will simply become more challenging: our data volumes, variety, and velocity will continue to grow. Novel, hard to reproduce solutions will continue to drive short-term differentiation. And increasing market and consumer complexity will drive an ongoing need for simplification. While the title will likely change, what won't are the core skills. And given the importance of data in driving differentiation, neither will the demand for data scientists, regardless of what they're called.

\section{Acting on Insight to Create Value: The Value Architect KEY POINTS:}

- Value architects hold skills parallel to but different from data scientists and help through managing change and linking insight to value

- The best value architects draw their experience from change management, strategic planning, and operational analytics

- Value architects are usually organically developed rather than formally trained and are best sourced through recommendations Data scientists are important. However, on their own they're not the answer. A common mistake is to assume that by draining the market of data scientists, organizations will assure themselves of long-term differentiation.

Most who follow this path simply find themselves throwing good money after bad. Data scientists are an essential part of extracting insight from information. Acting on that insight to derive value requires skills that most data scientists lack.

Like the contrary forces of yin and yang, data scientists need their counterpart if they're to create value. Insight without action is less than worthless; usually, it creates confusion and drives dissent. Left to their own devices and given appropriate support, most data scientists will create more answers than most organizations know to handle.

The answer isn't to constrain or limit data scientists; doing so will make them look elsewhere. It is better to make sure their genius is channeled towards driving change. Working out the probabilities of a winning hand in blackjack given the cards that have already been played is straightforward to a trained statistician. However, it usually takes someone with a different mentality to use that knowledge to make a living out of counting cards.

These "theotors", literally the gods of twist, are experts in change. Almost "actipreneurs", they are experts in making sure insights are acted on to drive real benefits. As value architects, their focus is less on the insight and more on the importance of designing systems, culture, and a passion for creating value through business analytics.

Communication is critical to a value architect. However, their skills go well beyond this: they move beyond being a communicator to being a true change agent, one that understands how to define, persuade, and drive organizational transformation. The heart of business analytics is change - every insight suggests a better way to do business. It is only when that change is made real that the business benefits. And getting the organization to that point requires more than just a good answer.

Making change happen involves justifying a need for change. Value architects do this in three ways. Firstly, they have a keen understanding of what the change is worth. Secondly, they understand how the change will make the organization's world better. And finally, they understand how to make the change real.

Change just for the sake of change usually destroys value. Most of us have been through one or more organizational transformations that, while well intentioned, actually made it harder to do business. A good change agent is able to explicitly link the change that's needed to real returns. Value architects are experts in translating the insights generated by data scientists into measurable benefits. Acting on customer sentiment insights might improve net promoter scores. Understanding market sentiment might improve market

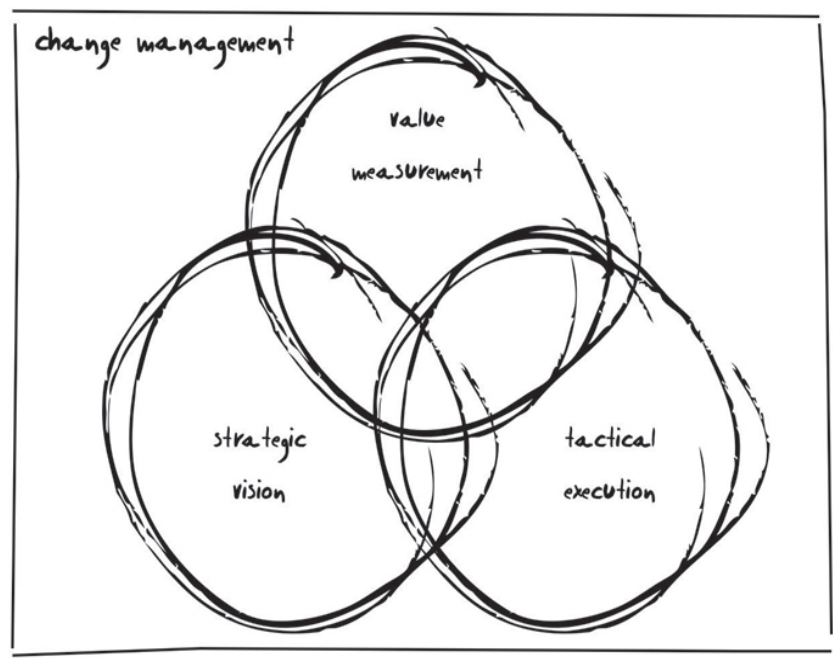


pricing. Good value architects are able to link these insights to measurable business benefits.

Until delivered, these linkages are theoretical. An effective value architect takes the steps needed to create operational processes that act on insight. It's only when insight is acted upon that organizations realize value. Value architects ensure that insights are acted on.

Benefits without vision are transitory. Value architects are experts in linking short-term returns to long-term competitive differentiation. Being able to link customer responses to churn rates might help improve short-term profitability. Using customer and market sentiment to drive a contextually relevant and true one-toone customer relationship, on the other hand, drives differentiation. In addition to translating the worth of tactical delivery, value architects help link business analytics to strategic differentiation. And, more importantly, they understand how to translate tactical improvements into strategic gains.

Value architects are less common than data scientists. Their experience comes from operational analytics, change management, and strategic planning. Given that most data scientists are focused on data, detail, and mathematical creativity, it's rare to find one person who has both sets of skills. Unfortunately, without someone to evangelize and drive real change, the investment most organizations make in data scientists goes to waste. Insight without action adds, not reduces, cost.

This resourcing problem is unlikely to go away in the near-term; the best value architects draw from hard-won experience. While it's possible to train someone with the raw technical skills needed to become a data scientist (if not the sheer curiosity), it is almost impossible to train a value architect. Sourcing external talent usually involves recommendations and a demonstrated history of success. In the absence of this, however, most organizations need to plan to develop and nurture these skills through direct experience.

\section{The Labor Market: Trends and Threats}

Across value architects and data scientists, three trends are clear:

- Demand for their skill sets will continue to rise across industries

- Action and relevancy will only become more essential

- Retention will be the differentiator

Much of the labor market shortage is due to the prevalence of big data. Every industry from retail to banking to government is facing a variety of big data challenges. This presents a unique opportunity for skilled resources: the ability to broaden their experience and salary through "industry-hopping".

What's good for the person is not always good for the organiza- tion. In theory, this ability to draw on other industries for resources broadens the traditional labor market. For industries without a strong history of deep analytics such as retail, recruiting from other industries can actually be an advantage. However, the disadvantage it brings is significant: given information asymmetries and the general labor market shortage, labor mobility creates an even bigger supply-side problem. There simply aren't enough people to go around. With demand increasing everywhere, everyone struggles.

Typically, graduate inflows would offset over-demand. Unfortunately, the hard reality is that while graduates fill a specific set of market demand, they also lack a key skill: business knowledge and application relevancy. While strong in raw skills, their commercial knowledge is usually lacking. Informally, some organizations have even gone so far as to say that, "it takes us a few years to un-train all the bad habits graduates were taught at school!"

This isn't because graduates are incompetent. It's because many of the problems organizations face are outside the scope of most higher education programs. Opportunities to work with big data, operational analytics, and change management are available to a very small set of elite programs. And, without this experience, many graduates are forced to start from first principles.

Finally, retention will rapidly become one of the biggest differentiators. Industries that have not historically been data or customer insight-driven are now starting to wake up. Even worse, many of these organizations are very large with vast untapped demand. This is putting significant competitive pressure on hiring effective data scientists and value architects. Similarly, consultancies such as dunnhumby, Precima, Accenture, McKinsey, and Deloitte are attracting and training top talent. Salaries are rising and will continue to rise in the future.

Hiring top talent is only the start. If an organization can't retain that talent, they lose more than just the person. They lose organizational knowledge and "know-how". In a market characterized by data knowledge and information advantage, it can be difficult to recover from this loss.

\section{Finding and Attracting the Right People}

Starting the search involves external and internal preparation. Finding the best talent involves connecting with the community. If you have the network, it's simply a case of putting out feelers. If you don't have the network, it is simply a case of talking to someone who does. Once found, the talent needs a reason to join. Without having the essentials in-place internally, bringing in high-quality talent becomes that much harder.

In parallel to your search, it's worth working on three things: 
- Cleaning up your data

- Clarifying your vision

- Committing to the strategy

Talented people rarely enjoy tedious activity. Instead, they enjoy finding insights and discovering trends; they don't want to be spending their time on tedious jobs like cleaning data.

Without the right tools, they are likely to start re-considering their acceptance. Be willing to invest in proper infrastructure. Without the right toolkit, a data scientist is just a frustrated genius. And, without the systems to support translating insights into action, the best a value architect can do is get consensus, not outcomes.

Start automating "uninteresting" work. The best data scientists are problem solvers, not "business as usual" people. Use technology to automate as much data preparation and cleansing work as possible, thus freeing up people to allow them to focus on business problems (the more rewarding aspect of their role).

Finally, clarify your vision. Make sure you understand your value proposition as a team and company. Without a strong strategy to draw from, data scientists and value architects will be acting in a vacuum.

In a skills-scarce marketplace, candidates have no shortage of opportunities. If they benchmark appropriately, most companies offer comparative salaries, bonuses, and career progression. Therefore, a key differentiator can be when the hiring manager is highly regarded in the market, is approachable, and provides a work environment that offers interesting problems.

Having a consultant who takes the time to understand your business, your requirements and, probably most importantly, your culture is paramount to the successful construction of any high achieving analytic team. A critical part of this involves being realistic with salaries, packages, incentives and benchmarking appropriately.

\section{Getting Started: The Hiring Checklist}

In starting your search for data scientists and value architects, it's useful to consider the following checklist:

- Where are they? The best resources may not be in your city let alone your country. Opening your search to a global market can uncover skills you might not have known even existed.

- What are they interested in? Creating an attractive value proposition does not always mean offering more money. While salaries need to be globally competitive, many skilled people are interested in more than just money. They may be interested in the opportunity to "create" something new or transform the way a company operates. Knowing what is available elsewhere helps refine your offer and make it more competitive, sometimes even with less remuneration.
- What are they going to do? Gaining clarity on your business analytics strategy can ensure your new recruits will hit the ground running. The strategy you develop will ensure your organization is able to take immediate advantage of the expertise of the new recruits, while avoiding the confusion and delays often caused by ad hoc recruitment.

\section{- What are they going to work with? Without technology and} high-quality data, data scientists and value architects have nothing to work with. When budgeting, make sure you have money set aside not just for resources but also for the tools they'll need. Engage early with vendors to understand the true cost of ownership; making assumptions without direct experience can create untenable situations.

Once your direction is clear, it is important to act. Every moment spent delaying is another wasted opportunity.

\section{Sidebars \\ The Players}

In every business analytics project there are three parties that need to agree. While it's the data scientist's job to come up with the answer, it's the value architect's job to get everyone to the table and agree on the right path forward.

These groups are:

- The business analytics team

- The business

- The information technology team

The business analytics team is where the data scientist and value architect live. Their primary responsibility is using data to create insight.

Without the right context, their answers are likely to be incorrect. It's the role of the business to bring domain knowledge to the group, providing guidance as to what makes a recommendation effective or ineffective.

The business has responsibility for the outcome: without their commitment, nothing will happen.

Finally, the information technology team is responsible for creating well-governed and highly available systems. They "own" the data, the systems, and the processes that create operational systems.

All three parties have a critical part to play in driving better outcomes through business analytics. Without the business analytics team, the organization lacks the technical skills to translate data into actionable insight. Without the business, it's impossible to gain commitment to change. And, without information technology, it's impossible to create highly automated processes that improve operational decision-making. 


\section{The Goal}

Business analytics might seem to be about insight. With titles like "data scientist" and "statistician", it's an easy mistake to make.

Business analytics isn't really about insight. It's about change management. And once organizations realize that, it makes all the difference.

Information offers a potential advantage. By analyzing detailed information, organizations can make better decisions. By definition, that analysis ends up suggesting new ways to run the business and new decision-making processes.

That insight alone is worthless. It's only when the organization acts on that insight and changes the way it does business that it can realize the value of analytics. And that involves change.

Change is challenging. While it's easy to come up with an answer, it's usually hard to convince someone that that answer's the right one. It's even harder to convince them that they should behave differently because of that answer.

It's for that reason that the most important capability in business analytics isn't analytics. It's about understanding how to encourage, manage, and drive change.

\section{Getting Hired}

Whether you're interested in becoming a data scientist or a value architect, it's helpful to focus on five areas:

- Technical Skills: When it comes to working with data, deep mathematics, statistics and computer science skills are all essential. If your analytical skills are rusty, look for ways to sharpen them.

- Teamwork Skills: Working well in a team is critical. Regardless of whether one's a data scientist or a value architect, being truly effective inevitably involves working closely with the business. This is key to teasing out business problems and moving quickly to workable solutions.

\section{- Communication and Influencing Skills: It's easy to think it's} all about presentation skills and the ability to structure a wellreasoned argument. More important is the ability to build trust between analysts and the business. Change comes from being able to communicate in a credible way with decision makers such that they trust what they are being told. Often, this involves trials or tests to prove or demonstrate the value of the insight.

- Business Knowledge: To be effective, analysts need to have a sound contextual understanding of what the business is about so that they can bring that contextual understanding to the data analysis. The only way to do this is to become close to the business.
- Tool Mastery: Complex software tools are critical to analyzing massive amounts of data. There are a great many analytical tools on the market and partnerships with these companies is key to ensure that the analysts make the most of the tools at their disposal and stay abreast of emerging tools and techniques.

\section{Hiring the Right Person}

Once the role is defined, and the vision cleared, and once HR understands that data scientists and value architects are specialist roles and do not entirely fit into standard salary 'bands', all that's left is to hire the right person.

Posting ads online and hoping for the best talent to apply rarely works. In many situations, "posting and praying" can have a strike rate of less than $2 \%$ for every application submitted. The better starting point is through your network. Engage search agencies that specialize in Big Data Analytics, understand your pain points, culture, and vision.

As your search progresses, make sure you allocate sufficient time to interview candidates. Without appropriate time management, holidays and other priorities can make the recruitment process drag on. Eventually, the vacancy becomes stagnant and candidates become reluctant to apply for an apparently undesirable role. Experience is also critical. Make sure to involve a good panel of interviewers spanning business to technology to the data experts with a focus on validating their quantitative skills, their programming skills, and their communication skills.

Given the relative newness of the role, many experienced data scientists and value architects come from an experience-based rather than trained background. Because their skills will be aligned to their experience, it is important to plan for targeted training and development. Someone who is a great culture fit, and has analytical capabilities and value measurement knowledge but lacks certain programming skills may need to get retrained in-house.

Being prepared to accept someone that doesn't have every skill needed (supported by an appropriate training program) is a pragmatic approach. Going to market with an unrealistic list of skills inevitably leads to disappointment. Companies that attract the best talent tend be flexible with their approach and requirements list.

The following have been shown repeatedly to be a useful checklist of capabilities to look for:

- Can they identify the business problem / challenge and how they might use data to solve the problem?

- Can they communicate their efforts and results to the business team? 
- Can they look at numbers and derive meaningful business insights that can be actioned?

When it comes to more technical capabilities, the following elements form a useful litmus test:

- Has 10 years experience of data mining / predictive analytics

- Has excellent programming and / or technical skills in how to create analytical datasets (such as a single view of customer)

- Has developed and applied a variety of predictive models (common examples include response and retention models) for a number of business domain areas
- Has developed and applied segmentation systems where use of advanced statistical and non statistical techniques have both been deployed or employed

- Has conducted numerous data analytics projects both strategic and non-strategic in nature. Has been able to create a disciplined measurement and tracking framework within organizations where he / she has worked.

- Has current leadership responsibilities managing a team of people accountable for the above activities. Case studies and worked examples are always useful. As part of the interview process, working on a standard / hypothetical project can be used as a pressure test.

\section{References:}

1. IDC. (2011) 2011 Digital Universe Study: Extracting Value from Chaos. Retrieved March 6, 2013, from http://www.emc.com/collateral/demos/microsites/emc-digital-universe-2011/index.htm

2. Manyika, J., Chui, M., Brown, B., Bughin, J., Dobbs, R., Roxburgh, C., \& Byers, A.H. (2011) Big Data: The next frontier for innovation, competition, and productivity. McKinsey Global Institute. Retrieved March 6, 2013, fromhttp://www.mckinsey.com/lnsights/MGI/Research/ Technology_and_Innovation/Big_data_The_next_frontier_for_innovation

3. Gartner. (2012) Gartner Says Big Data Creates Big Jobs. Retrieved March 6, 2013, from http://www.gartner.com/newsroom/id/2207915

4. Economist Intelligence Unit. (2011) Big data: Harnessing a game-changing asset. The Economist. Retrieved March 6, 2013 from http://www.sas.com/resources/asset/SAS_BigData_final.pdf

5. Tweeted by Joel Cherkis on 21/10/12: https://twitter.com/jcherkis/status/260093078004707328

6. Manyika, J., Chui, M., Brown, B., Bughin, J., Dobbs, R., Roxburgh, C., \& Byers, A.H. (2011) Big Data: The next frontier for innovation, competition, and productivity. McKinsey Global Institute. Retrieved March 6, 2013, from http://www.mckinsey.com/lnsights/MGl/Research/ Technology_and_Innovation/Big_data_The_next_frontier_for_innovation

7. TechAmerica. (2013). Big Data and the Public Sector. Retrieved March 6, 2013, from http://www.techamericafoundation.org/content/ wp-content/uploads/2013/02/SAP-Public-Sector-Big-Data-Report_FINAL-2.pdf

8. Stubbs, E. Delivering Business Analytics: Practical Guidelines for Best Practice. Hoboken, NJ: John Wiley \& Sons, 2011.

9. Kiron, D., Ferguson, R.B., \& Prentice, P.K. (2013) From Value to Vision: Reimagining the Possible with Data Analytics. MIT Sloan Management Review

10. Box, J.F. (1987) Guinness, Gosset, Fisher, and Small Samples. Statistical Science, Vol 2., No. 1, 45-52. Retrieved March 6, 2013 from http://www2.fiu.edu/ blissl/GuinessGossetFisher.pdf

11. Stubbs. E. (2011) The value of business analytics. Analytics Magazine. Retrieved March 6, 2013 from http://www.analytics-magazine.org/ special-articles/461-the-value-of-business-analytics

12. Patil, D.J. (2011) Building Data Science Teams. O’Reilly. Retrieved March 6, 2013 from http://radar.oreilly.com/2011/09/ building-data-science-teams.html

13. Stubbs, E. The Value of Business Analytics: Identifying the Path to Profitability. Hoboken, NJ: John Wiley \& Sons, 2011

14. Davenport, T.H., Harris, J.G., \& Morison, R. Analytics at Work: Smarter Decisions, Better Results. Cambridge, MA: Harvard Business Review Press, 2010.

Evan Stubbs is the author of The Value of Business Analytics: Identifying the Path to Profitability and the book Delivering Business Analytics: Practical Guidelines for Best Practice, both published by Wiley Press. His next book, focusing on driving innovation, is tentatively scheduled for March 2014. He has been published in various magazines including Analytics Magazine and sascom. His practical and experience-based talks on creating value through the use of business analytics are in high demand.

Chami Akmeemana is the Founder and Managing Director of Huntel Global a search firm that specializes in Business Analytics recruitment. Prior to establishing Huntel Global, Chami owned and managed multiple successful business ventures in Australia. Before moving to Australia in 2005, Chami worked with various corporate and government bodies in the UK and was also a British Police officer where he worked in undercover policing. He holds a Masters in Bioceramic Engineering. 